\title{
Chondrosarcoma in the Petrous Apex: Case Report and Review
}
F. Banaz ${ }^{1}$
I. Edem ${ }^{2,4}$
I. D. Moldovan 2,5
S. Kilty ${ }^{1,4,5}$
G. Jansen ${ }^{3,4}$
F. Alkherayf $f^{2,4,5}$

\begin{abstract}
Address for correspondence Fahad AlKherayf, MD, MSc, CIP, FRCSC, Division of Neurosurgery, Ottawa Hospital-Civic Campus, 1053 Carling Avenue, Room C2218, Ottawa, Ontario, K1Y 4E9, Canada (e-mail: falkherayf@toh.ca).
\end{abstract} Hospital, Ottawa, Canada

${ }^{3}$ Department of Pathology and Laboratory Medicine, The Ottawa Hospital, Ottawa, Canada

4 University of Ottawa, Faculty of Medicine, Ottawa, Canada

${ }^{5}$ The Ottawa Hospital Research Institute, Ottawa, Canada

J Neurol Surg Rep 2018;79:e83-e87.

\section{Introduction}

Chondrosarcomas are a heterogeneous group of malignant tumors formed by tumoral cells that produce cartilage matrix. ${ }^{1}$ The majority of skull base chondrosarcomas are low grade and exhibit an indolent growth pattern. ${ }^{2}$ Almost all skull base chondrosarcomas arise in synchondroses near the temporooccipital junction ${ }^{3}$. Also, the vast majority of chondrosarcomas are sporadic without known risk factors. However, a small proportion occurs in the context of Maffucci's syndrome or Ollier's disease. ${ }^{4}$ Given their usually slow growth rate, they are capable of reaching sizable dimensions, promoting bone

received

April 11, 2018

accepted after revision

August 28, 2018
DOI https://doi.org/

10.1055/s-0038-1673627. ISSN 2193-6358. erosion, and significant displacement of neurovascular structures before causing symptomatology that will eventually lead to diagnosis. Cranial neuropathies and headaches are common complaints on presentation. ${ }^{5}$

Rosenberg et al (1999) ${ }^{6}$ reported on 200 patients who were treated at the Massachusetts General Hospital (Boston) for skull base chondrosarcomas and noted the following site distribution: temporo-occipital $66 \%$, clivus $28 \%$, and sphenoethmoid complex $6 \%$.

Computed tomography (CT) and magnetic resonance imaging (MRI) are employed to evaluate the tumor on
License terms

Stuttgart · New York

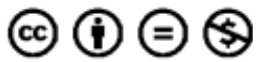


presentation. CT generally shows a lytic lesion and is used to examine the extent of bone invasion and tumor mineralization. ${ }^{7}$ The lesion can be seen to erode local bone and there is usually heterogeneous enhancement on contrast administration, although enhancement can be variable. ${ }^{8}$ On CT scan, the majority of these tumors will show a well-defined zone of transition with normal adjacent bone. Calcification is present in approximately $50 \%$ of tumors, typically in rings and arcs. ${ }^{8}$ MRI is useful to demonstrate the soft tissue extent of the tumor $^{2}$ and to delineate the extent of the intraosseous and soft tissue involvement preoperatively. ${ }^{1}$

Transnasal routes to the medial petrous apex have been reported $^{9-11}$ and are gaining popularity with the advent of endoscopic approaches. These approaches are particularly useful when the lesion has created a window by expanding the petrous apex medially toward the clivus. In these cases, the petrous apex remodels protrudes into the sphenoidal sinus along the lateral sphenoid recess allowing the lesion to intrude medially into the sphenoidal sinus beyond the genu of the internal carotid artery (ICA) and providing direct access via the sphenoidal sinus. ${ }^{12}$ Despite local aggressiveness, these lesions are often spare the dura, compressing, and displacing rather than transgressing it. ${ }^{5}$

\section{Case Report}

Our patient was a 57-year-old female who was presented to clinic with acute onset of left isolated abducens (cranial nerve
VI) palsy and occasional headache mainly on the left side of the retro-orbital area with some radiation to the left occiput. On physical exam, she had a left VI nerve palsy but no other neurological deficits or complaints. The cranial nerve examination was otherwise normal as language and gait testing. CT angiogram of the brain and neck, during admission, was showed a lytic lesion at the left petrous apex and left clivus with cortical breakthrough measuring approximately $18 \times 11.7 \mathrm{~mm}$. Patient's MRI T1 with gadolinium contrast and T2 images revealed hyperintense, heterogeneously enhancing, well-defined mass in the left petrous apex measuring $2.4 \times 1.8 \times 1.9 \mathrm{~cm}($ AP $\times$ transverse $\times$ craniocaudal $)$ with extension to the adjacent clivus ( - Fig. 1 ). The mass breached the bony cortex in the left prepontine and premedullary region. Results of a metastatic workup were negative.

The case was discussed in the multidisciplinary skull base meeting. It was decided to access the tumor using the extended endoscopic endonasal transclival approach to the left petrous apex and to reconstruct the defect with a pedicled nasoseptal flap, using frameless stereotactic navigation and neuromonitoring as adjuncts.

Intraoperative frozen section diagnosis was matched the final histopathological diagnosis of chondrosarcoma. Microscopic images showed islands with chondroid matrix including lacunae with slightly atypical chondrocytes having slightly enlarged hyperchromatic nuclei. There was a sparse fibrovascular stroma and no mitotic activity. At the margins of these islands the tumor showed a myxoid change of the matrix with
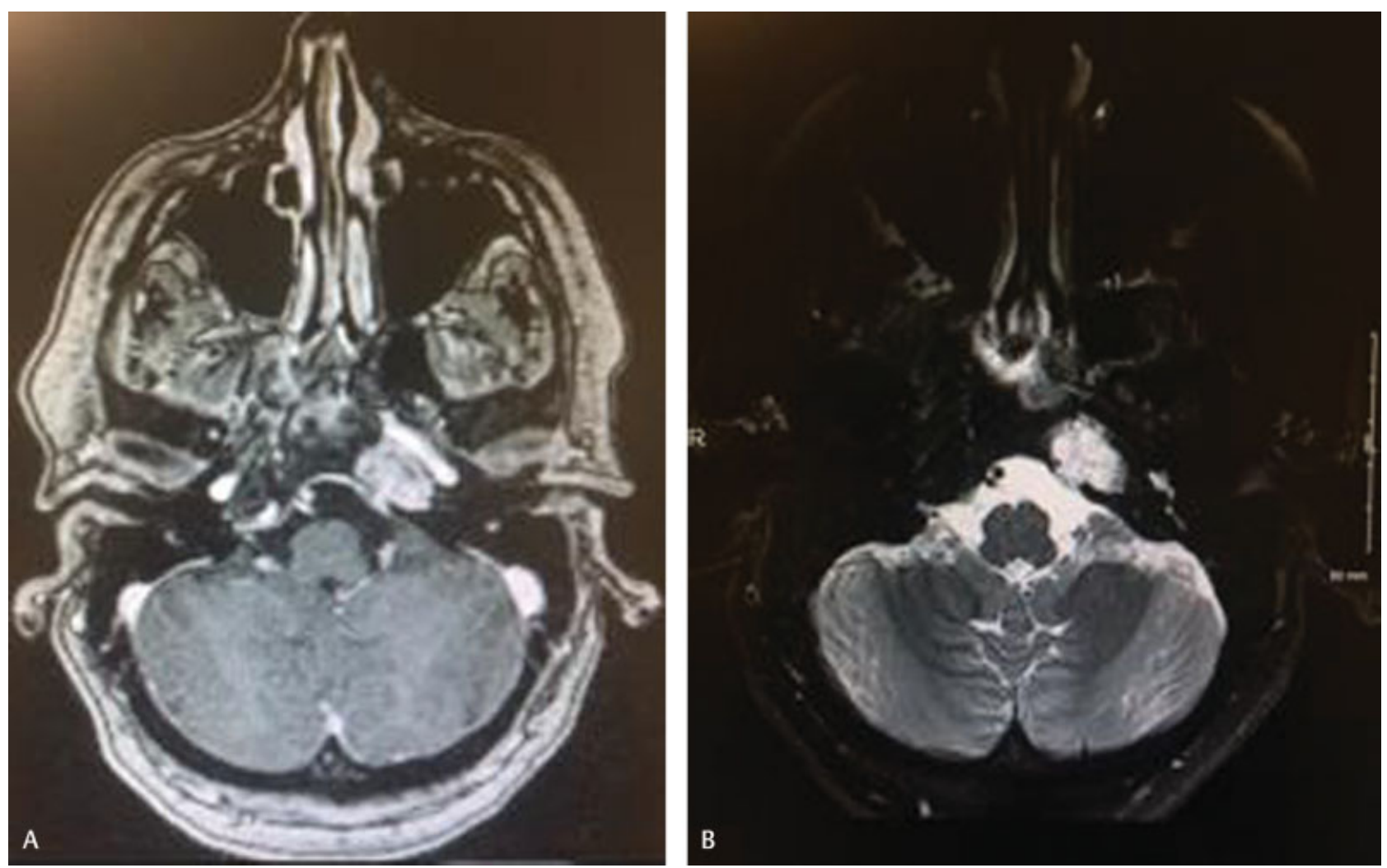

Fig. 1 Preoperative MRI images of left petrous apex lesion. (A) MRI T1 with gadolinium contrast and (B) T2 images revealed hyperintense, heterogeneously enhancing, well-defined mass in the left petrous apex measuring $2.4 \times 1.8 \times 1.9 \mathrm{~cm}$ (AP $x$ transverse $x$ craniocaudal), with extension to the adjacent clivus. AP, anteroposterior; MRI, magnetic resonance imaging. 


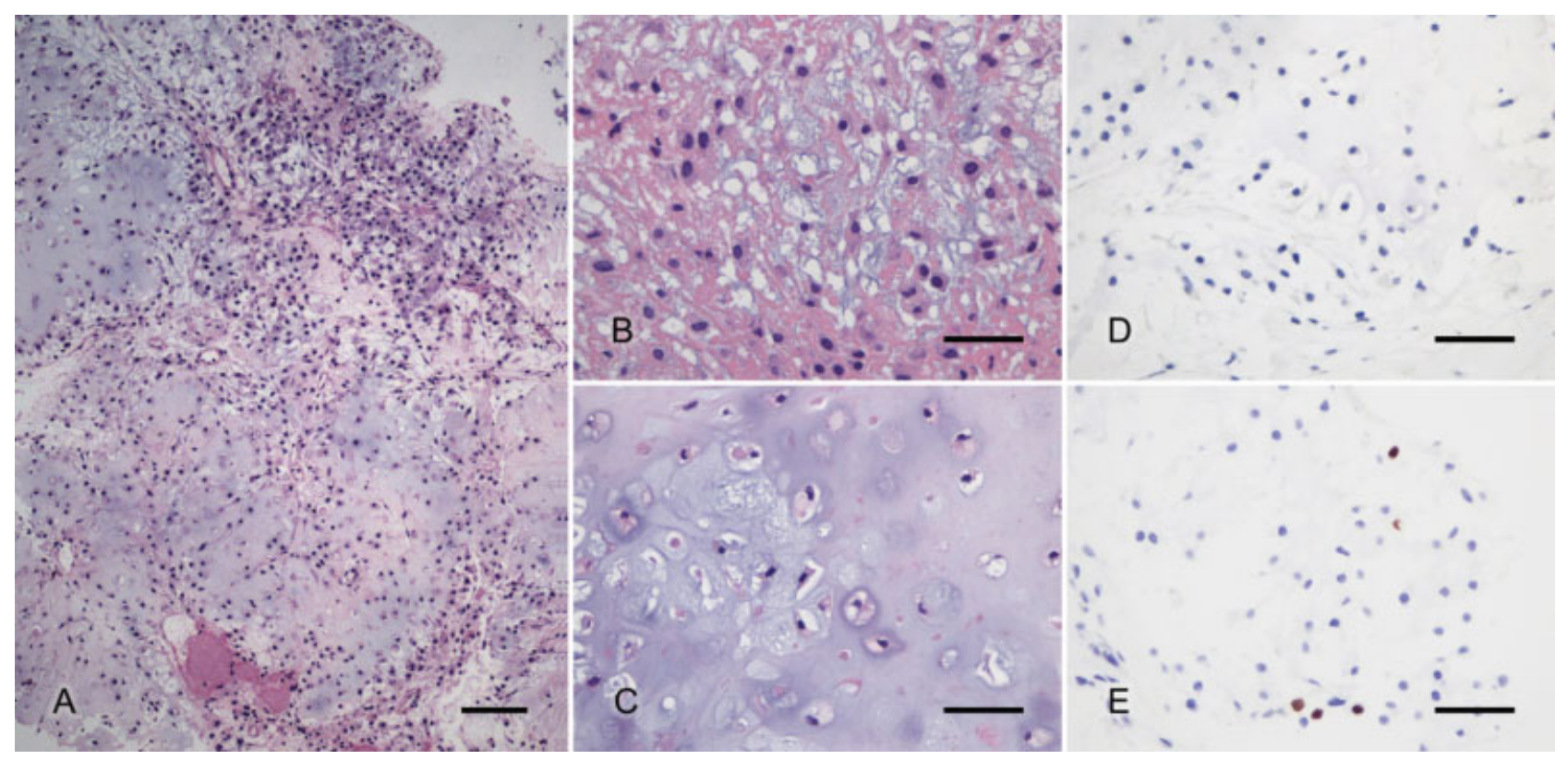

Fig. 2 Histopathology test results. (A) Overview showing islands of chondroid matrix surrounded by fibrovascular matrix and higher grade areas with higher cellularity with myxoid change of the matrix (H\&E); (B) Detailed higher grade area (H\&E); (C) Detailed lower grade area (H\&E); (D) Absence of pan cytokeratin staining (cocktail Millipores AE1/3 and Leica CK8/CK18); (E) Proliferation in selected hypercellular and myxoid areas of the tumor (MIB-1 Dako). Bar is $100 \mu \mathrm{m}$ in $\mathrm{A}$, and $50 \mu \mathrm{m}$ in $\mathrm{B}-\mathrm{E}$. H\&E, hematoxylin and eosin. AE, anti-cytokeratin monoclonal antibodies; MIB, mouse lgG binding protein; CK, cytokeratin.

an increase in cellularity. No mitoses were observed. There was no expression of wide spectrum cytokeratins at immunohistochemistry testing. MIB-1 (mouse IgG binding protein) proliferation rate varied between 2 and $5 \%$. These findings are consistent with an intermediate grade chondrosarcoma ${ }^{13}$ (-Fig. 2).

The surgical procedure was uncomplicated. The VI nerve palsy remained stable and no new deficits occurred. The patient was discharged home on postoperative day 4. Postoperative MRI axial and Sagittal T1 post contrast showed no residual tumor on the left petrous bone (-Fig. $\mathbf{3}$ ).

\section{Operative Technical Notes}

The extended endoscopic endonasal approach (EEA) is most useful for chondrosarcomas, chordomas, and sinonasal lesions of the petroclival space. The approach may prove to offer an advantage for these medially positioned lesions by providing a direct route to the lesion without the need for a lateral approach, minimizing the need for neurovascular manipulation. ${ }^{12}$ Indeed, the potential advantage of EEA for petrous apex chondrosarcomas includes the possibility of accessing multiple skull base compartments, even bilaterally, in a single procedure while also avoiding retraction or manipulation of neurovascular structures. ${ }^{5}$

The procedure began with a reduction of the right inferior turbinate and then a right middle turbinectomy. Then, a retrograde uncinectomy followed by a type 3 maxillary antrostomy was performed. The bullae ethmoidalis were opened out to the lamina papyracea and the anterior and posterior ethmoidectomies were completed. The sphenoid ostium was identified and opened to allow for a sphenoidotomy. The right sided pedicled nasoseptal flap ${ }^{14}$ was then harvested to be used at the end of the surgery for reconstruction and was stored in the right maxillary antrum. A posterior septectomy was performed to allow for binarial access to the sphenoid sinus. The left sphenoid os was identified and opened followed by excision of the septum down to the posterior wall of the sinus. The sphenoid mucosa was removed and the rostrum drilled down to then was allowed drilling of the sinus floor.

With neuronavigation guidance, the location of the paraclival carotid arteries was verified and the left petrous carotid was identified. The sella was drilled down to the beginning of the clivus and then the clivus between the carotids was drilled out. The clival resection was stopped short at the dura keeping the inner cortex intact. This wide and extended transclival approach provided substantial access to the left petrous apex of the temporal bone. The course of the carotid arteries was also confirmed with Doppler ultrasound sonography and neurophysiologic monitoring occurred throughout the case. The left petrous apexectomy with removal of the tumor was then occurred. An incision of the tumor capsule was performed and an intraoperative specimen was sent for pathologic analysis and was consistent with chondrosarcoma. Then, careful internal debulking of the lesion in a piecemeal fashion was performed. Dissection of the tumor capsule was then followed using a set of dissectors that enable direct motor stimulation with live feedback from the neuromonitoring team to avoid damage to the cranial nerves. Chondrosacromas in the petous apex have been reported to be usually soft and amenable to suction, yet very hard and calcified ones can also be encountered. ${ }^{5}$ Once complete tumor resection was achieved including removal of infiltrated bone, copious irrigation of the operative field with warm saline was done. This step promoted hemostasis and removal of any debris. ${ }^{5}$

We did not encounter any intraoperative cerebrospinal fluid (CSF) leak. The right sided pedicled nasoseptal flap was used to cover the defects in the clivus and right petrous apex 

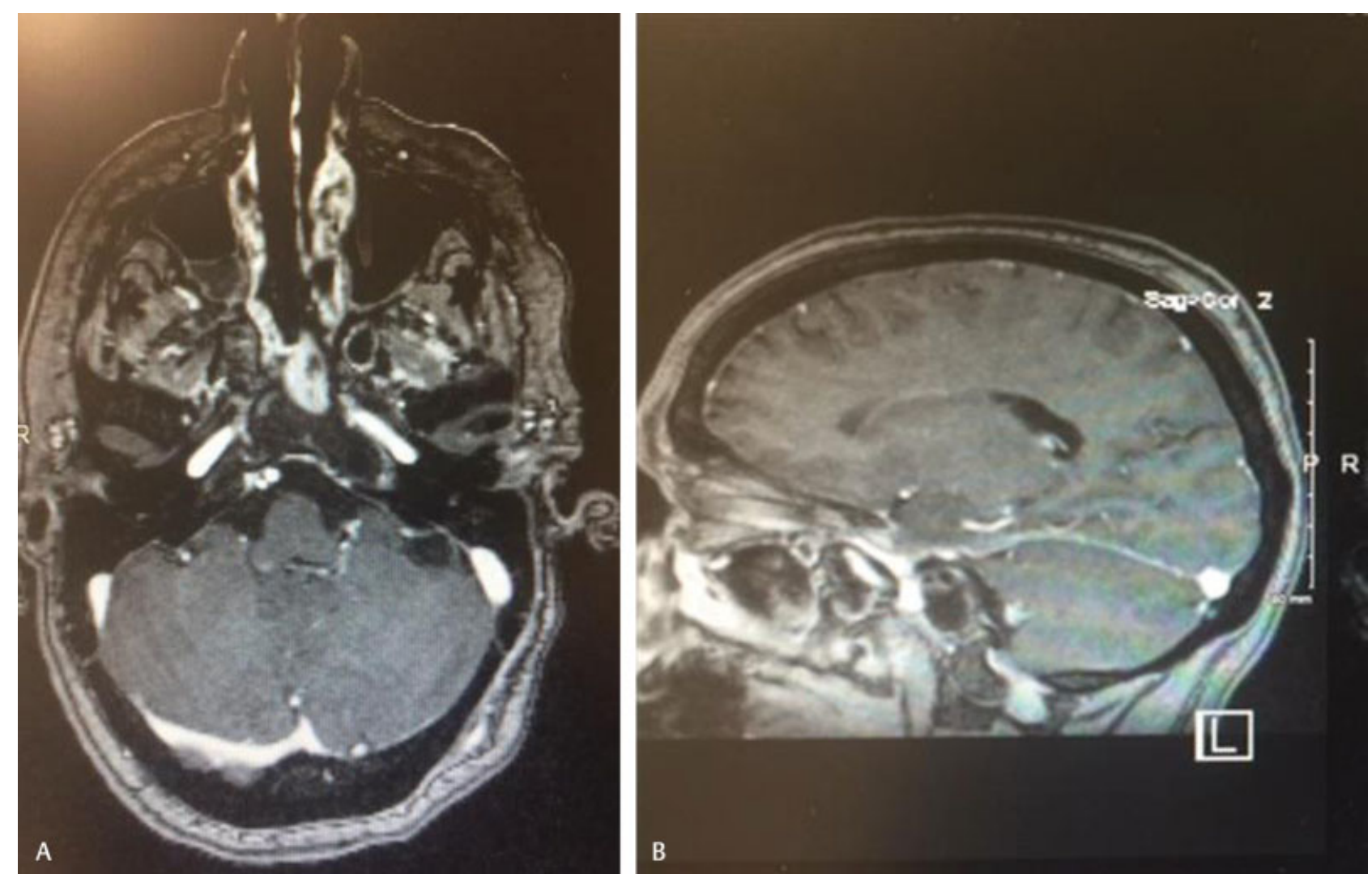

Fig. 3 Postoperative MRI images of left petrous apex lesion. Postoperative (A) MRI axial and (B) Sagittal T1 post contrast images showed no clear residual area of abnormal enhancement on the left petrous bone. MRI, magnetic resonance imaging.

as well as the exposed dura. Damp and crushed Gelfoam were then placed around the graft and fibrin glue (Tissel) placed around its perimeter. The surgery was finalized by obliteration of the sphenoid and ethmoid sinuses with Gelfoam and the placement of bilateral septal splints and then Merocel nasal packs coated with antibiotic ointment. The recovery and postoperative period was uneventful.

\section{Discussion}

In our case, we performed a one-stage procedure to remove a petrous apex chondrosarcoma using an expanded endoscopic endonasal transclival approach. This has been seen in other studies including Mesquita Filho et al study $(2014)^{15}$ who reported five cases with petrous apex chondrosarcomas removed using an endoscopic endonasal resection without any reported postoperative complications. Also, another group of authors were performed one-stage expanded EEAs and skull base reconstruction with the use of a navigation system to successfully treat two cases with skull base chondrosarcomas associated with Maffucci's Syndrome. ${ }^{16}$

On the other hand, Crockard et al (2001) reported 17 histologically verified skull base chondrosarcoma cases, 14 of which had a tumor located at the petrous apex and clivus requiring sequential procedures that were staged between 3 weeks and 3 months apart. The approaches used included posterior fossa exposures, pterional, transfrontal, total pet- rosectomy, and open-door maxillotomies. There was one noted postoperative death that occurred within the first 28 postoperative days in a patient diagnosed with a massive petrous apex chondrosarcoma that was removed using a combined transtemporal-extradural middle fossa approach. The patient was died of a pulmonary embolus. There were two CSF leaks, each occurring during a mastoidectomy, which were difficult to control and required several procedures to treat including lumbar drains. New cranial nerve deficits developed in four patients as a result of the surgery and one patient who had undergone previous surgery and radiotherapy suffered a wound breakdown that required a secondary procedure for closure. Samii et al $(2009)^{17}$ presented similar results with $25 \%$ of 18 operated patients developing new postoperative cranial nerve deficits and $55 \%$ of those with preoperative deficits not improving. Traditionally, this had been achieved by piecemeal surgical resection. However, this type of resection has been associated with a local recurrence rate of $53 \%{ }^{1}$

Given the high morbidity with radical surgery for extensive skull base tumors, low complete cure rates with surgery alone and the indolent growth pattern, some would advocate for a shift in the goal of surgical resection. ${ }^{14}$ The development of radiologic imaging with early diagnosis and microsurgical/endoscopic skull base approaches might have favored better short-term results in these recently reported cases. $^{16}$ 


\section{Prognosis and Outcome}

Bloch and colleagues $(2009)^{18}$ stated that prognosis is primarily based on multiple factors. The first factor is the extent of tumor resection. ${ }^{18}$ The second factor is the tumor histological grade. ${ }^{18}$ Grade I and II tumors have a better prognosis than Grade III tumors. The World Health Organization (WHO) classification recognizes three grades of chondrosarcomas. WHO Grade I is well differentiated and low grade. Cellularity is higher than with enchondroma but with occasional plump nuclei. The classic histological subtype falls into this category and is the most common type seen in the skull base (approximately $90 \%$ ) and in more elderly patients ( $60-70$ years). WHO Grade II is moderately differentiated and intermediate grade. There is increased cellularity and distinct nucleoli are seen in the majority of cells. WHO Grade III is poorly differentiated and high grade. These are highly malignant lesions histologically characterized by high cellularity, prominent nuclear atypia, and the presence of mitosis. They are usually seen more in younger patients (20-40 years) and have a higher tendency for recurrence. ${ }^{8}$ In their review, Maslehaty et al. found that $50 \%$ of all skull base chondrosarcoma lesions were WHO Grade I tumors and $90 \%$ were WHO Grades I and II collectively. ${ }^{19}$ The predominance of the low-grade types within the skull base gives this condition its overall good prognosis. Grade III tumors are rare in the skull base and are associated with a worse prognosis. ${ }^{20,21}$ Finally, adjuvant radiotherapy has been associated with a survival benefit. ${ }^{18}$

\section{Conclusion}

Aiming for maximal tumor resection with function preservation, the choice of surgical approach and grade of resection must be carefully assessed and should be tailored differentially to each patient, while also considering the experience and preference of the surgical team. Complete resection is the treatment of choice when the tumor is amenable to total removal.

Extended endoscopic endonasal transclival approach to the petrous apex with reconstruction appears to be a safe and feasible technique, capable of achieving total or near total resection in most cases with identified lesions at the petrous apex. Nonetheless, future studies with a greater number of patients are crucial to confirm and consolidate this initial finding.

Funding

There was no funding for this case report.

\section{Conflicts of Interest}

None.

\section{Authors' Contributions}

All authors have contributed to manuscript preparation and review.

F.B. and I.E. did literature review.

G.J. did pathological examination of surgical specimen.

\section{References}

1 Gelderblom H, Hogendoorn PC, Dijkstra SD, et al. The clinical approach towards chondrosarcoma. Oncologist 2008;13(03): 320-329

2 Kim MJ, Cho KJ, Ayala AG, Ro JY. Chondrosarcoma: with updates on molecular genetics. Sarcoma 2011;2011:405-437

3 Raghu M, Moumoulidis I, De R, Moffat D. Chondrosarcomas of the temporal bone: presentation and management. J Laryngol Otol 2004;118(07):551-555

4 Bloch O, Parsa AT. Skull base chondrosarcoma: evidence-based treatment paradigms. Neurosurg Clin N Am 2013;24(01):89-96

5 Ditzel Filho LF, Prevedello DM, Dolci RL, et al. The endoscopic endonasal approach for removal of petroclival chondrosarcomas. Neurosurg Clin N Am 2015;26(03):453-462

6 Rosenberg AEMD, Nielsen GP, Keel SB, et al. Chondrosarcoma of the base of the skull: a clinicopathologic study of 200 cases with emphasis on its distinction from chordoma. Am J Surg Pathol 1999;23(11):1370-1378

7 Crockard HA, Cheeseman A, Steel T, et al. A multidisciplinary team approach to skull base chondrosarcomas. J Neurosurg 2001;95 (02):184-189

8 Awad M, Gogos AJ, Kaye AH. Skull base chondrosarcoma. J Clin Neurosci 2016;24:1-5

9 Eisenberg MB, Haddad G, Al-Mefty O. Petrous apex cholesterol granulomas: evolution and management. J Neurosurg 1997;86 (05):822-829

10 Pontius AT, Ducic Y. Extended orbitozygomatic approach to the skull base to improve access to the cavernous sinus and optic chiasm. Otolaryngol Head Neck Surg 2004;130(05):519-525

11 Sekhar LN, Burgess J, Akin O. Anatomical study of the cavernous sinus emphasizing operative approaches and related vascular and neural reconstruction. Neurosurgery 1987;21(06):806-816

12 Kassam AB, Gardner P, Snyderman C, Mintz A, Carrau R. Expanded endonasal approach: fully endoscopic, completely transnasal approach to the middle third of the clivus, petrous bone, middle cranial fossa, and infratemporal fossa. Neurosurg Focus 2005;19 (01):E6

13 Purgina B, Lai CK. Distinctive head and neck bone and soft tissue neoplasms. Surg Pathol Clin 2017;10(01):223-279

14 Kassam AB, Thomas A, Carrau RL, et al. Endoscopic reconstruction of the cranial base using a pedicled nasoseptal flap. Neurosurgery 2008;63(1, Suppl 1):ONS44-ONS52, discussion ONS52-ONS53

15 Mesquita Filho PM, Ditzel Filho LF, Prevedello DM, et al. Endoscopic endonasal surgical management of chondrosarcomas with cerebellopontine angle extension. Neurosurg Focus 2014; 37(04):E13

16 Beer-Furlan A, Balsalobre L, Vellutini EA, Stamm AC. Endoscopic endonasal approach in skull base chondrosarcoma associated with Maffucci syndrome: case series and literature review. World Neurosurg 2016;85:365.e7-365.e15

17 Samii A, Gerganov V, Herold C, Gharabaghi A, Hayashi N, Samii M. Surgical treatment of skull base chondrosarcomas. Neurosurg Rev 2009;32(01):67-75, discussion 75

18 Bloch OG, Jian BJ, Yang I, et al. A systematic review of intracranial chondrosarcoma and survival. J Clin Neurosci 2009;16(12): 1547-1551

19 Maslehaty H, Petridis AK, Kinzel A, Binay Y, Scholz M. Chondrosarcoma of the petrous bone: a challenging clinical entity. Head Neck Oncol 2013;5(02):13

20 Gay E, Sekhar LN, Rubinstein E, et al. Chordomas and chondrosarcomas of the cranial base: results and follow-up of 60 patients. Neurosurgery 1995;36(05):887-896, discussion 896-897[discussion 896-7]

21 Kaye AH, Laws ER. Brain tumors: an encyclopedic approach. 3rd ed. Edinburgh/New York: Saunders/Elsevier; 2012 